\title{
SOLUTION OF NONLINEAR EQUATIONS AND COMPUTATION OF MULTIPLE SOLUTIONS OF A SIMPLE REACTION-DIFFUSION EQUATION
}

\author{
ADRIAN SWIFT $^{l}$ and EASWARAN BALAKRISHNAN ${ }^{2}$
}

(Received 16 March 1998)

\begin{abstract}
The first part of this paper starts with a brief discussion of some methods for solution of nonlinear equations which have interested the first author over the last twenty years or so. In the second part we discuss a recent research involvement, the success of which relies heavily on the numerical solution of nonlinear equation systems. We briefly describe path-following methods and then present an application to a simple steady-state reactiondiffusion equation arising in combustion theory. Results for some regular geometric shapes are shown and compared with those from an approximate method.
\end{abstract}

\section{Solution of nonlinear equation systems}

The main thrust of this paper is to discuss some methods for the solution of nonlinear equation systems containing parameters applied to a specific physical model. That particular system may be written as

$$
\mathbf{F}(\mathbf{u}, \mu)=\mathbf{0},
$$

where $\mathbf{u} \in \mathfrak{R}^{n}$ and $\mu \in \mathfrak{R}^{m}$.

We commence with a brief discussion of Newton-like methods which have interested the first author over many years. These methods are described in the context of solving the non-parametric equations

$$
\mathbf{F}(\mathbf{x})=\mathbf{0},
$$

where $\mathbf{F}, \mathbf{x} \in \mathfrak{R}^{n}$.

\footnotetext{
'Department of Mathematics, Massey University, Albany, New Zealand.

${ }^{2}$ Department of Mathematics, Massey University, Palmerston North, New Zealand.

(C) Australian Mathematical Society 2000, Serial-fee code $0334-2700 / 00$
} 
All Newton-type methods assume that an initial approximation $\mathbf{x}^{0}$ to a solution $\mathbf{x}^{*}$ is given. After $k$ steps we assume that an approximation $\mathbf{x}^{k}$ has been reached. At this step a direction $\mathbf{d}^{k}$ is sought, and $\mathbf{F}(\mathbf{x})=\mathbf{0}$ linearized about $\mathbf{x}^{k}$ so that, if $\mathbf{J}\left(\mathbf{x}^{k}\right)$ is the Jacobian of $\mathbf{F}\left(\mathbf{x}^{k}\right)$, then

$$
\mathbf{F}\left(\mathbf{x}^{k}+\mathbf{d}^{k}\right)=\mathbf{F}\left(\mathbf{x}^{k}\right)+\mathbf{J}\left(\mathbf{x}^{k}\right) \mathbf{d}^{k}+O\left(\left\|\mathbf{d}^{k}\right\|^{2}\right) .
$$

1.1. Newton method The classical Newton method [8] truncates the above equation after the linear term so that the direction $\mathbf{d}^{k}$ is found by solving the linear equation system

$$
\mathbf{J}\left(\mathbf{x}^{k}\right) \mathbf{d}^{k}=-\mathbf{F}\left(\mathbf{x}^{k}\right)
$$

and then setting $x^{k+1}=x^{k}+d^{k}$. A line search, with line search parameter, $\alpha^{k}$, is often incorporated, in which case $x^{k+1}=x^{k}+\alpha^{k} d^{k}$.

1.2. Discrete Newton method Since the basic Newton method requires evaluation of the analytic Jacobian matrix at each iteration, it is rarely used in that form, though modern computer algebra software has made this process less arduous. Traditionally, some modification is incorporated to reduce the overall computational effort. Much software which incorporates gradient information computes this approximation to the Jacobian using finite-difference approximations [8]. A popular choice is the forwarddifference approximation to column $j$ of $\mathbf{J}\left(\mathbf{x}^{k}\right)$, namely

$$
\frac{\mathbf{F}\left(\mathbf{x}^{k}+h_{j} \mathbf{e}_{j}\right)-\mathbf{F}\left(\mathbf{x}^{k}\right)}{h_{j}},
$$

where $\mathbf{e}_{j}$ is column $j$ of the unit matrix I. The resultant method is referred to as the discrete Newton (DN) method. Choice of the $h_{j}$ is a balance between discretization and rounding errors, and is commonly taken as the square root of machine epsilon $\left(\sim 10^{-8}\right.$ for double precision (64-bit) IEEE arithmetic) in a relative sense. This variant is also time consuming in that $n$ additional function evaluations are required for each iteration.

1.3. Shamanskii variation The Shamanskii [17] modification fixes the Jacobian at a given value for $m$ steps, choosing $m$ to maximize some efficiency index. The efficiency index used by Brent [4] is the logarithm of that used by Ostrowski, and Brent shows that it has the value

$$
E=\frac{\ln (m+1)}{n+m}
$$

The method can introduce a significant reduction in computational effort if $m$ is chosen to maximise $E$. For $n=10$, for example, $m=7$ and $E \approx 0.122$. Since the efficiency index of DN is $\ln 2 /(n+1) \approx 0.063$, the Shamanskii modification is nearly twice as efficient as the basic DN method. 
1.4. Brown-type methods A completely different approach to modifying Newton's method was introduced by Brown [5]. Rather than linearize all components of $\mathbf{F}(\mathbf{x})$ simultaneously, as is done in the straight Newton method, the component equations are linearized sequentially. Let the $j^{\text {th }}$ partial derivative of $F_{i}(\mathbf{x})$ be defined by

$$
F_{i, j}^{k}=\frac{\partial F_{i}\left(\mathbf{x}^{k}\right)}{\partial x_{j}} .
$$

Assuming that the first component, $F_{1}(\mathbf{x})$, of $\mathbf{F}(\mathbf{x})$ is the first to be linearised, it can be rearranged to solve for the component $x_{r}$ of $\mathbf{x}$ for which the partial derivative is the largest in magnitude $\left(\left|F_{1, r}^{k}\right| \geq\left|F_{1, j}^{k}\right|\right)$. This gives

$$
x_{r}=x_{r}^{k}-\sum_{j \neq r}^{n} \frac{F_{1, j}^{k}}{F_{1, r}^{k}}\left(x_{j}-x_{j}^{k}\right)-\frac{F_{1}^{k}}{F_{1, r}^{k}} .
$$

Then the second component $F_{2}(\mathbf{x})$ of $\mathbf{F}(\mathbf{x})$ is linearized, and $x_{r}$ substituted so that $F_{2}(\mathbf{x})$ is a function of the remaining $n-1$ variables. This process is repeated, $n-1$ times in all. Overall, each iteration requires only $1 / 2$ of the function evaluations of the discrete Newton method. However, at each stage a back substitution is required to find the relevant $F_{j}(\mathbf{x})$, so that considerable computational effort may be required here. Brown's original implementation (based on $L U$ factorizations), required $O\left(n^{4}\right)$ flops, but Gay [13] was able to reduce this to $O\left(n^{3}\right)$. Brent produced a variant based on $Q R$ factorizations, also requiring $O\left(n^{3}\right)$ flops.

The previously mentioned Shamanskii modification can be incorporated into either the Gay or Brent methods. Joe [14] performed tests which showed that the corresponding Gay implementation appeared to be more efficient and robust than that applied to the method of Brent.

1.5. Broyden (quasi-Newton) methods Broyden [6] avoided the problems of direct computation of the Jacobian, or a discrete approximation to it, by using a simpler approximation to it (or its inverse). This method can be very effective if the starting vector is sufficiently close to the sought solution. Such methods may be viewed as variants on the generalised secant method.

1.6. Continuation (homotopy) methods The main weakness of the methods described above is the desirability of providing a good starting vector $\mathbf{x}^{0}$. Davidenko [10] suggested an alternative strategy (in the context of integral equations) in which the original problem was modified by the introduction of a parameter $\theta \in[0,1]$ such that

$$
\mathbf{G}(\mathbf{x}, \theta)=\mathbf{F}(\mathbf{x})-\theta \mathbf{F}\left(\mathbf{x}^{0}\right) .
$$

The solution of $\mathbf{G}(\mathbf{x}, 0)=\mathbf{0}$ is the required solution of the equation system $\mathbf{F}(\mathbf{x})=\mathbf{0}$. With a suitable strategy for computing the sequence of $\theta$ values, starting with $\theta=1$, 
Broyden [7] demonstrated that this method can be very effective for solving systems of nonlinear equations. In the univariate case, Broyden's method [6] reduces to the conventional secant method, and Swift and Lindfield [18] described an effective continuation method based on this approach (this algorithm is the basis for the NAG routine C05 AJF).

\section{Path following}

Any of the methods described in Section 1 may be used to solve the algebraic system

$$
\mathbf{F}(\mathbf{u}, \mu)=\mathbf{0}
$$

for fixed values of the parameters $\mu$. In many such problems, it is desirable to find the range of values of the parameters for which a solution exists. We assume that the solution for a particular parameter $\lambda$ is desired, all other parameters in $\mu$ being fixed (see Eilbeck [11] for example). Some starting point $\mathbf{u}_{0}=\mathbf{u}\left(\lambda_{0}\right)$ is assumed to be given and we assume also that the method has progressed to find $\mathbf{u}(\lambda)$ for the particular $\lambda$. Then the implicit function theorem ensures that $\mathbf{u}^{\prime}(\lambda)=\mathrm{du}(\lambda) / \mathrm{d} \lambda$ is found by solving the equation

$$
\mathbf{F}_{\mathbf{u}}(\mathbf{u}(\lambda), \lambda) \mathbf{u}^{\prime}(\lambda)+\mathbf{F}_{\lambda}(\mathbf{u}(\lambda), \lambda)=\mathbf{0} .
$$

To extend the solution to $\lambda+\delta \lambda$, find an initial estimate $\mathbf{u}^{0}(\lambda+\delta \lambda)$ of the solution using an Euler method so that

$$
\mathbf{u}^{0}(\lambda+\delta \lambda)=\mathbf{u}(\lambda)+\delta \lambda \mathbf{u}^{\prime}(\lambda) .
$$

A Newton (or Newton-like) corrector is used to improve this initial approximation, the correction $\delta \mathbf{u}^{k}$ to $\mathbf{u}^{k}, k=1,2, \ldots$, satisfying the nonlinear system

$$
\mathbf{F}_{\mathbf{u}}\left(\mathbf{u}^{k}, \lambda+\delta \lambda\right) \delta \mathbf{u}^{k}+\mathbf{F}_{\lambda}\left(\mathbf{u}^{k}, \lambda+\delta \lambda\right)=\mathbf{0} .
$$

The solutions may be used to plot some measure of the solution $\mathbf{u}$, such as $\|\mathbf{u}\|_{\infty}$, as a function of $\lambda$. In most such situations, the researcher wishes to investigate bifurcation and other critical points at which the Jacobian becomes singular, so that Newton's method will fail. To overcome this, Keller [15] suggested the introduction of an additional parameter $s$ (the pseudo-arc length), which requires an additional normalisation equation, so that the expanded system has the form

$$
\mathbf{F}(\mathbf{u}(s), \lambda(s), s)=\mathbf{0}, \quad \mathbf{N}(\mathbf{u}(s), \lambda(s), s)=\mathbf{0} .
$$

The path-following algorithm described above is then applied to these $n+1$ nonlinear equations, with $s$ as the path-following parameter rather than $\lambda$. 


\section{Reaction-diffusion equation}

In this section we briefly describe a combustion model whose critical parameters may be computed by setting up an approximating nonlinear equation system.

Suppose we have a body of potentially combustible material undergoing exothermic reaction in a bounded region $\Omega$. Neglecting reactant consumption, and assuming Arrhenius kinetics, the equation for heat balance can be written (see Wake and Jackson [19] for example)

$$
k \nabla^{2} T+\sigma Q A \exp \left(-\frac{E}{R T}\right)=c \frac{\partial T}{\partial t} .
$$

On the boundary $\partial \Omega$, the most general boundary condition will be

$$
k \frac{\partial T}{\partial n}+H\left(T-T_{a}\right)=0 .
$$

An explanation of the various physical and chemical parameters appearing in these equations may be found in the paper of Balakrishnan, Swift and Wake [1].

3.1. Steady state formulation We are particularly interested in the behaviour of the steady-state solution with increasing ambient temperature $T_{a}$. In this case the reaction-diffusion equation is

$$
k \nabla^{2} T+\sigma Q A \exp \left(-\frac{E}{R T}\right)=0 .
$$

3.2. Frank-Kamenetskii formulation The original approach was due to FrankKamenetskii [12] (the "old" variable case) which took $\theta=E\left(T-T_{a}\right) / R T_{a}^{2}$, the dimensionless temperature rise over ambient, as the dependent variable.

Under the Frank-Kamenetskii approximation, $\left(R T_{a} / E\right) \ll 1$, the steady-state heat balance equation simplifies to the "old" case equation

$$
\nabla^{2} \theta+\delta \exp (\theta)=0
$$

with boundary condition

$$
\frac{\partial \theta}{\partial n}+B i \theta=0
$$

The Frank-Kamenetskii parameter is given by $\delta=\sigma Q a_{0}^{2} E A /\left(k R T_{a}^{2}\right)$ and the Biot number by $B i=H a_{0} / k$, where $a_{0}$ is the half-width of $\Omega$.

Note that $T_{a}$ appears in the variable $\theta$, the parameter $\delta$ and the boundary condition, which makes direct computation of its critical values difficult. 
3.3. "New" variable formulation Motivated by the above-mentioned difficulty, Burnell, Graham-Eagle, Gray and Wake [9] introduced the "new" case, with dimensionless variable $u$ and dimensionless parameter $U$,

$$
u=\frac{R T}{E} \quad \text { and } \quad U=\frac{R T_{a}}{E} .
$$

In this case the governing steady-state heat balance equation has the form

$$
\nabla^{2} u+\lambda \exp \left(-\frac{1}{u}\right)=0
$$

with boundary conditions

$$
\frac{\partial u}{\partial n}+B i(u-U)=0 .
$$

Note that no approximations have been made here, unlike the "old" case. Hence, in either the "old" or "new" case, the model can be described by the equation

$$
\nabla^{2} u+\lambda f(u)=0
$$

where

$$
f(u)=\exp (u) \text { and } f(u)=\exp \left(-\frac{1}{u}\right),
$$

with boundary conditions

$$
\frac{\partial u}{\partial n}+B i u=0 \text { and } \frac{\partial u}{\partial n}+B i(u-U)=0,
$$

respectively. The bifurcation (distinguished) parameter in the "old" case is $\lambda$. In the "new" case it can be either $U$ or $\lambda$. However, in the reaction-diffusion problem being discussed, we are mainly interested in solution behaviour with ambient temperature, so take $U \propto T_{a}$ as the distinguished parameter, with $\lambda$ fixed.

\section{Shape factor method}

For regular shapes (class $A$ shapes, $N=1,2,3$, the infinite slab, infinite circular cylinder and sphere respectively) (1) has the one-dimensional form

$$
\frac{d^{2} u}{d r^{2}}+\left(\frac{N-1}{r}\right) \frac{d u}{d r}+\lambda f(u)=0, \quad 0<r<1,
$$

and so is not difficult to solve. Critical values found from the shape factor method for Class $A$ shapes will be accurate, assuming the boundary value solver is of suitable accuracy. Boddington, Gray and Harvey [3] generalised this ease of solution to other shapes by defining a shape factor for a body of volume $V$ and surface area $S$ in terms of an arbitrary dimension $N$ related to the geometry. Critical values found from this approach for non-Class $A$ shapes will be approximate only. 
4.1. Multiplicity of solutions One interesting feature of either form of the above steady-state equation is the existence of multiple solutions [1]. In the "old" variable case, when

$$
\lambda=(N-1) \exp \left(-\frac{2}{B i}\right),
$$

there is an infinite number of solutions for $N$ in the range

$$
2<N<10 \text {, }
$$

whilst in the "new" variable case, for sufficiently large values of $\lambda$, solutions of multiplicity greater than three may occur for any $N$ in the range

$$
2<N<12 .
$$

\section{Direct method}

In order to find accurate values of the critical parameters for non-Class $A$ shapes, the reaction-diffusion equations (1) and (2) must be solved numerically. We use $O\left(h^{2}\right)$ finite-difference approximations to the derivatives in these for the simplest twodimensional geometry, the square of side length 2 , centred at the origin, corresponding to the infinite square rod (ISR). Note that, in fact, this geometry is most poorly approximated by the shape factor method. If the interval $[0,1]$ is divided into $M-1$ equal intervals (so that $h=1 /(M-1)$ ) and taking advantage of symmetry, the discretization produces a $M^{2} \times\left(M^{2}+1\right)$ nonlinear algebraic system of the form used in Section 1, written as

$$
\mathbf{F}(\mathbf{w}, v)=\mathbf{0} .
$$

The function $\mathbf{F}$ is written as $B \mathbf{w}+c(f(\mathbf{w})) v+D f(\mathbf{w})$, where $\mathbf{w}$ is the vector of discretized approximations to $\mathbf{u}$ and $v$ is the bifurcation parameter $\delta, U$ for the "old", "new" cases respectively, labelled as an additional variable $v=w_{M^{2}+1}$ (though fixed as far as the current boundary value solution is concerned). The matrix $B \in \mathfrak{R}^{M^{2} \times M^{2}}$ is block tridiagonal, $D$ is a diagonal matrix, null in the "old" case as boundary conditions do not involve the bifurcation parameter, and the vector $c \in \Re^{M^{2}}$. The software package PITCON [16] uses a form of Newton iteration to solve these equations and numerical evidence indicates that computed estimates of the critical parameters are accurate to $O\left(h^{2}\right)$. A similar procedure is used to solve the reaction-diffusion equation over a cube; in this case it is essential to choose the mesh size to provide a balance between excessive computation and accuracy. A mesh size of $h=0.1(M=11)$ turns out to give critical values accurate to within about $0.2 \%$ [2]. 


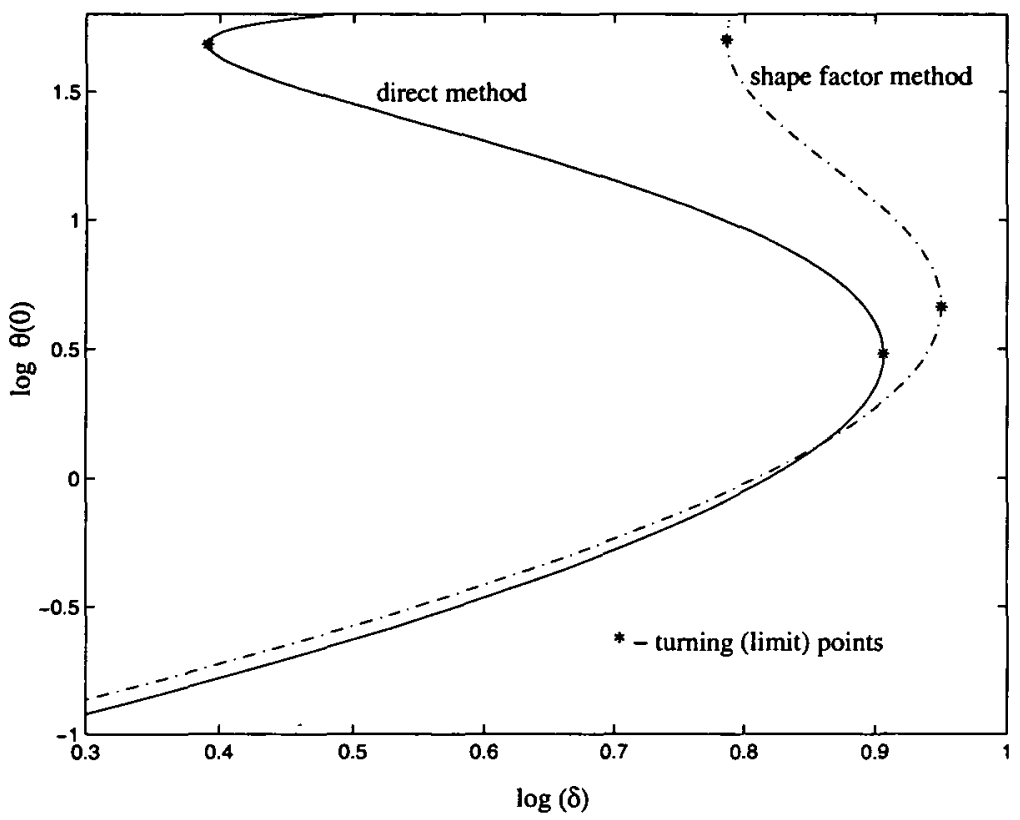

FIGURE 1. Comparison of methods-"old" variable.

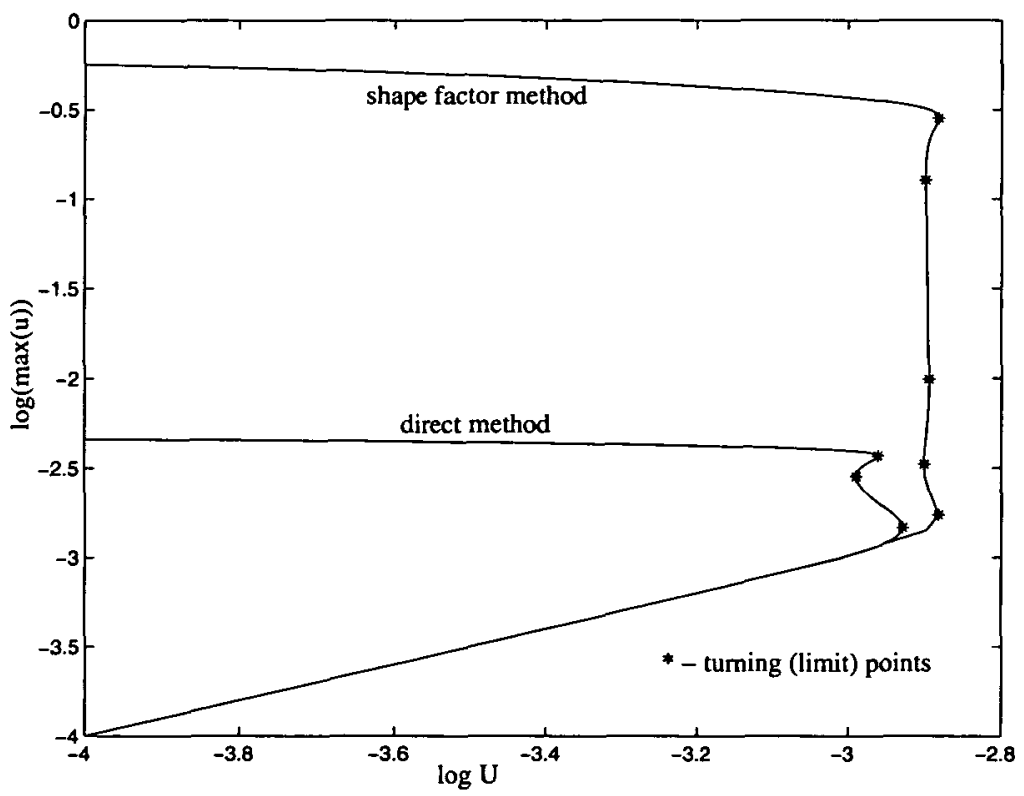

FIGURE 2. Comparison of methods-_"new" variable. 


\section{Results}

Our primary interest is the study of the solution set $(u, U, \lambda)$ for a unit ISR and a unit cube and we compare the effectiveness of the shape factor approach with the direct numerical approach in computing these solution sets. In a practical situation, interest lies in the onset of critical behaviour as the distinguished parameter increases from zero. The critical parameter values associated with this phenomenon are referred to as $\delta_{c r}$ in the "old" variable case and $U_{c r}$ in the "new" variable case. Note that $U_{c r}$ will exist only if $\lambda$ is greater than a certain critical $\lambda_{t r}$. Note also that $\delta_{c r}$ and $\lambda_{t r}$ correspond to ignition points in the "old" and "new" variable cases respectively. Both the methods given are implemented for two- and three-dimensional non-class $A$ shapes. Figure 1 shows a comparison of the shape factor and direct methods for a cube in the "old" variables, and Figure 2 shows a similar comparison in the "new" variables.

Tables 1 and 2 display the numerical values for the critical parameters found by each of the shape factor and direct methods. The direct method values are accurate to $0.2 \%$ as mentioned in Section 5 .

TABLE 1. Comparison of $\delta_{c r}$ values for the $\mathrm{e}^{\theta}$ case: $B i=\infty$.

\begin{tabular}{|l|c|c|c|}
\hline & Shape factor method & Direct method & \% difference \\
\hline ISR & 1.717 & 1.701 & 0.9 \\
\hline Cube & 2.586 & 2.475 & 4.5 \\
\hline
\end{tabular}

TABLE 2. Comparision of $\lambda_{t r}$ values for the $\mathrm{e}^{-(1 / u)}$ case: $B i=\infty$.

\begin{tabular}{|l|c|c|c|}
\hline & Shape factor method & Direct method & \% difference \\
\hline ISR & 9.577 & 9.341 & 2.5 \\
\hline Cube & 15.470 & 14.201 & 8.9 \\
\hline
\end{tabular}

\section{References}

[1] E. A. Balakrishnan, A. Swift and G. C. Wake, "Path-following for disjoint bifurcation problems arising in ignition theory", Math. Comput. Modelling 19 (1994) 9-15.

[2] E. A. Balakrishnan, A. Swift and G. C. Wake, "Critical values for some non Class $A$ geometries in thermal ignition theory", Math. Comput. Modelling 24 (1996) 1-10.

[3] T. Boddington, P. Gray and D. I. Harvey, "Thermal theory of spontaneous ignition: Criticality in bodies of arbitrary shape", Phil. Trans. Roy. Soc. A270 (1971) 467-506.

[4] R. P. Brent, "Some efficient algorithms for solving systems of nonlinear equations", SIAM. J. Numer. Anal. 10 (1973) 327-344. 
[5] K. M. Brown, "A quadratically convergent Newton-like method based on Gaussian elimination", SIAM J. Numer. Anal. 6 (1969) 560-569.

[6] C. G. Broyden, "A class of methods for solving nonlinear simultaneous equations", Math. Comp. 19 (1965) 577-593.

[7] C. G. Broyden, "A new method of solving nonlinear simultaneous equations", Comp. J. 12 (1969) 94-99.

[8] R. L. Burden and J. D. Faires, Numerical Analysis, 5th ed. (PWS, 1993).

[9] J. Burnell, J. Graham-Eagle, B. Gray and G. Wake, "Determination of critical ambient temperatures for thermal ignition", IMA J. App. Math. 42 (1989) 147-154.

[10] D. F. Davidenko, "On a new method of numerical solution of systems of nonlinear equations", Doklady Akad. Nauk SSSR 88 (1953) 601-602.

[11] J. C. Eilbeck, “The pseudo-spectral method", SIAM J. Sci. Stat. Comp. 7 (1986) 599-610.

[12] D. A. Frank-Kamenetskii, Diffusion and Heat Transfer in Chemical Kinetics (Princeton University Press, 1955).

[13] D. M. Gay, "Brown's method and some generalisations, with applications to minimization problems", Technical Report TR 75-225, Dept. of Computer Science, Cornell University, 1975.

[14] S. Joe, "A survey of numerical methods for solving simultaneous nonlinear equations", M. Sc. project, Massey University, 1982.

[15] H. B. Keller, "Numerical solution of bifurcation and nonlinear eigenvalue problems", in Applications of bifurcation theory (ed. P. H. Rabinowitz), (Academic Press, 1977) 359-384.

[16] W. Rheinboldt, Numerical analysis of parametrized equations (John Wiley and Sons, 1986).

[17] V. E. Shamanskii, “A modification of Newton's method", Ukrain. Mat. Zh. 19 (1967) 133-138.

[18] A. Swift and G. R. Lindfield, "Comparison of a continuation method with Brent's method for the numerical solution of a single nonlinear equation", Comp. J. 21 (1978) 359-362.

[19] G. C. Wake and F. H. Jackson, "The heat balance in spontaneous ignition", N.Z. J. of Science 19 (1976) 23-27. 